\title{
Mitigate Water Scarcity with Intervention
}

\author{
QuanHui Che ${ }^{1}$ \\ School of North China Electric Power University, Baoding 071000, China \\ 2218461873@qq.com
}

Keywords: Water scarcity; input-output system; neural network algorithm

\begin{abstract}
Water scarcity has emerged as a top-tier global issue for both environment and development. However, how to mitigate water scarcity is a real problem. We choose Shandong Province in China with heavily overloaded water as our research object., we propose an intervention plan concerning social consciousness, pollution control, water source and industry structure. With the intervention plan, we regard Shandong as an input-output system to project the future water availability, in which intervention plan is input and impact of the plan is output. The process of calculating the direct and interactive impact that plan brings is the key component of the system using neural network algorithm. Ultimately, we reach the conclusions that Shandong will become less susceptible to water scarcity and water won't become a critical issue in the future due to our prevention plans.
\end{abstract}

\section{Introduction}

Water scarcity challenges people around the world. There are two primary causes for water scarcity: physical scarcity and economic scarcity. To be specific, water scarcity problem is how to balance the supply and demand of water. Scarcer water creates new challenges for energy supply because coal, oil, gas, and electricity production can require massive amounts of freshwater. Global population growth and economic development suggest a future of increasing demand, competition, and cost for limited freshwater supplies. Currently, more than 650 million people lack access to clean water ${ }^{[1]}$. So water scarcity urgently need to be addressed

\section{The Intervention Plan}

A comprehensive intervention plan is identified to mitigate water scarcity in four areas, which exactly corresponds to the four sets of indicators.

Measure 1: Open up water source.

Opening up water source aims to improve the carrying capacity of water resources through constructing renewable utilization system of water resources. The government should construct regulation and storage reservoirs of reclaimed water using seasonal river and idle depression to perfect the reclaimed water deployment, transportation and recycling system.

Measure 2: Identify the magnitude of water issues ${ }^{[2]}$.

The energy of water is often overlooked, generally because water is seen as a free or low-cost resource. While water deserves to gain more recognition as an important development challenge through collecting data to calculate the financial and environmental costs of water not just nationally, but at provincial and municipal levels, as water resources vary significantly throughout the country.

Measure 3: Ramp up demand-side management practices and policies ${ }^{[3]}$.

In addition to mandated energy intensity reductions and water consumption limits, the government needs to strengthen integrated approaches that look at the link between water use and distribution, particularly in the construction and industrial sectors. Just as there can be a negative domino effect in the competition for industrial water use, there can also be a positive multiplier effect when all are managed together. 


\section{Measure 4: The whole process of water pollution control.}

As for the industry pollution control, the government should strict the provision of pollution emission standards gradually by setting reasonable milestones of water quality. As for the pollution control in rural areas, solving the environmental problems associated tightly with the society and personal life is the emphasis, such as direct sewage discharge and garbage piled up along the river problems.

\section{The Input-output System $\operatorname{Model}^{[4]}$}

To study the influence of an intervention plan on a region, we put forward a model to study the water scarcity problem which exists in the topic. To simplify the problem, we abstract Shandong Province as a simplified input-output system. Then we study the influence of an intervention plan by seeing into the outputs' impacts on a region's running system.

The flow chart of the model is shown in Figure 1.1:

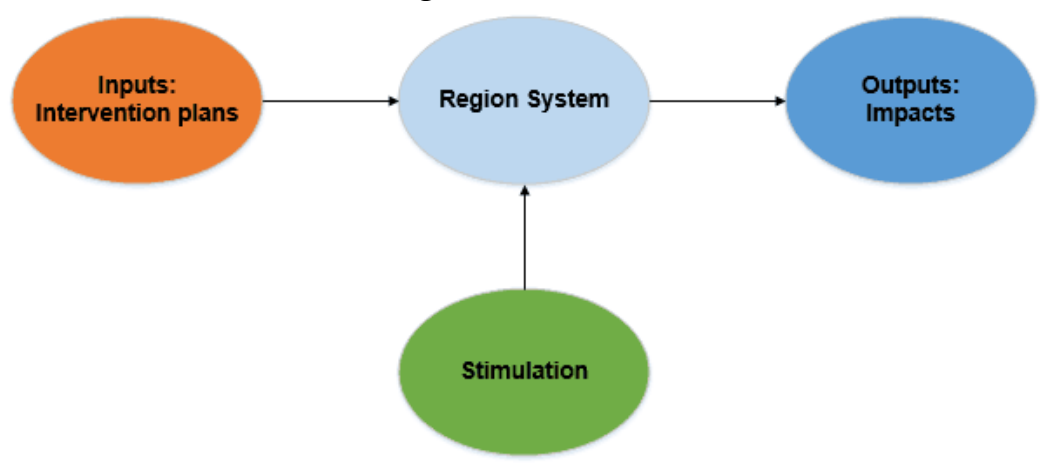

Figure 1.1

\section{The Evaluation of the Intervention Plan}

The intervention plan will affect the indicators in different degrees that weighing the water availability of a region. To determine the effects of additional intervention, we collect the data that the same or similar intervention plans bring to other region. Then by employing Neural Network Algorithm $^{[5]}$ to data mining, we obtain the intervention plans' effect on each indicator considered, which is shown in Figure 1.2.

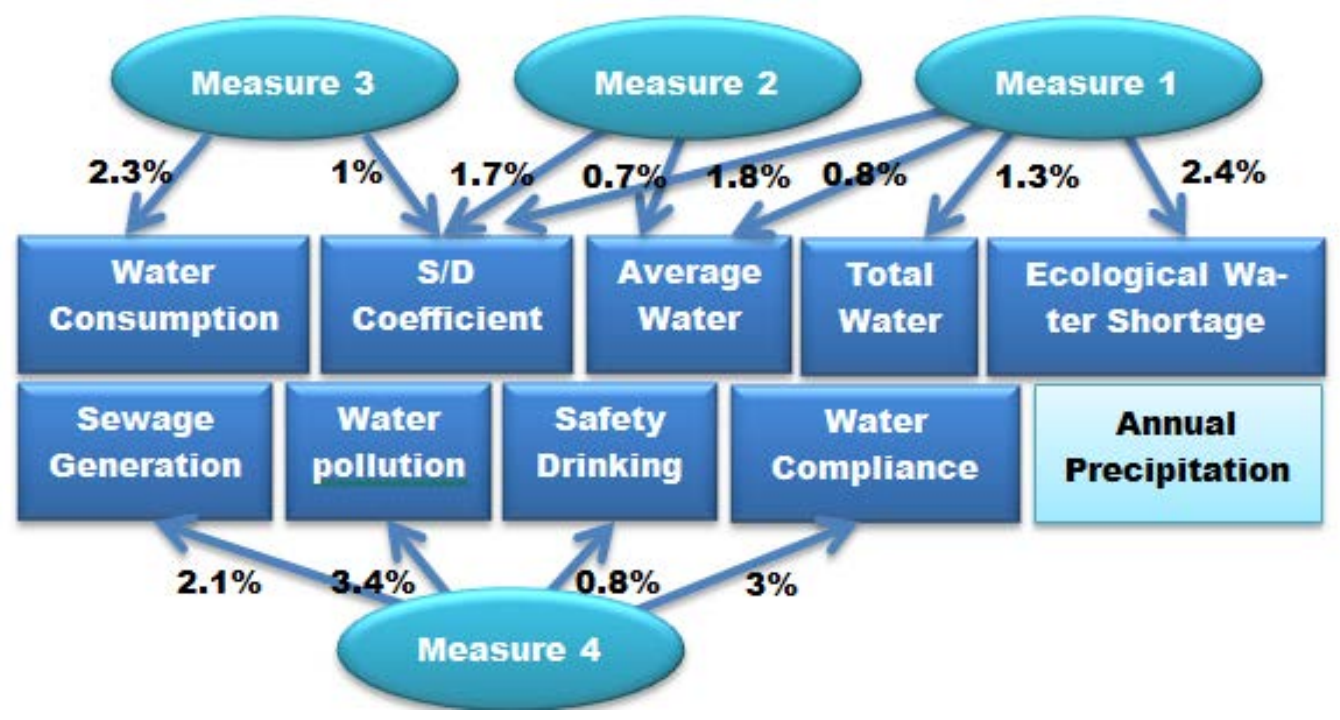

Figure 1.2The intervention plans' effect on each indicator 


\section{A Forecast on Shandong after Intervention Control}

After getting mutual influence between indicators and the intervention plan influence on each indicator, we apply the intervention plans proposed to our input-output system model and figure out the scores of system indicators and coordination indicators in each year using the same method as before. The result is clearly represented in Figure 1.3.
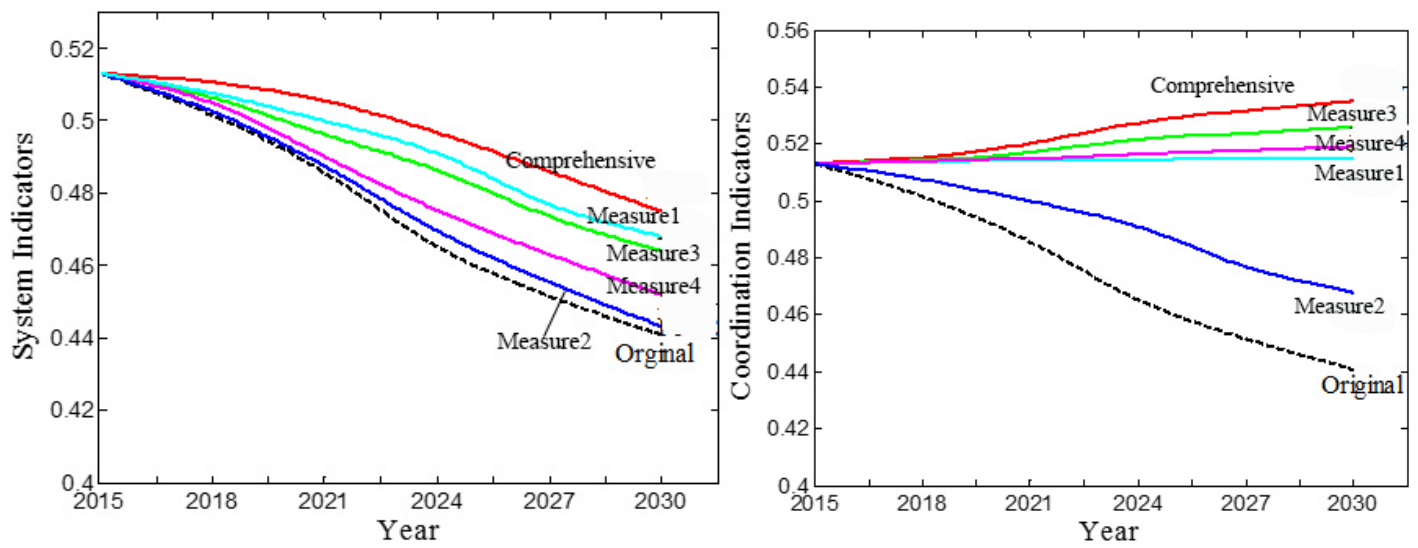

Figure 1.3the scores of indicators

Using the intervention we designed in Task 4, the comprehensive intervention plan containing four measures is obviously predominant than the other single-aspect measure. In terms of the environmental drivers, Measure 1 which aims to open up water source works better than other measures. As to the social drivers, Measure 3 that is committed to adjust the industrial structure works better.

Then we use the comprehensive level model to get the comprehensive score of Shandong in 15 years in Figure 1.4, from which we can know that the intervention plans make the water availability enhance expect Measure 2.

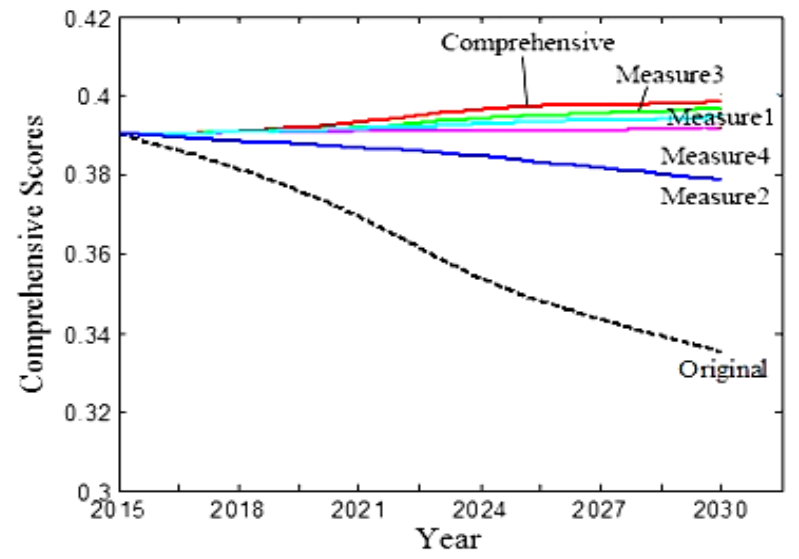

Figure 1.4 The comprehensive score

Due to the intervention plan, Shandong becomes less susceptible to water scarcity according to the increasing comprehensive scores. The intervention plan we propose will prevent water scarcity from being a critical issue.

\section{References}

[1] http://www.wri.org/blog/2016/01/solving-twin-crises-energy-and-water-scarcity

[2]The overall planning of water resources sustainable utilization of Shandong Province in early 21st century(2000 2030) http://www.docin.com/p-731164918.html

[3]http://www.newsecuritybeat.org/2015/03/chinas-water-energy-food-roadmap-global-choke-point 
-report/

[4] Shougui Si, Xi Sun. Mathematical modeling algorithm and application. Beijing: National Defence Industry Press, 2015.2. 358 359.

[5] Qiyuan Jiang, Jingxing Xie . Mathematical model. Beijing: Higher Education Press,2011. 